\title{
REVIEW
}

\section{Year in review 2009: Critical Care - cardiac arrest, trauma and disasters}

\author{
Jeffery C Metzger*, Alexander L Eastman and Paul E Pepe
}

\begin{abstract}
During 2009, Critical Care published nine papers on various aspects of resuscitation, prehospital medicine, trauma care and disaster response. One article demonstrated that children as young as 9 years of age can learn cardiopulmonary resuscitation (CPR) effectively, although, depending on their size, some may have difficulty performing it. Another paper showed that while there was a trend toward mild therapeutic hypothermia reducing S-100 levels, there was no statistically significant change. Another predictor study also showed a strong link between acute kidney injury and neurologic outcome while another article described a program in which kidneys were harvested from cardiac arrest patients and showed an $89 \%$ graft survival rate. One experimental investigation indicated that when a pump-less interventional lung assist device is present, leaving the device open (unclamped) while performing CPR has no harmful effects on mean arterial pressures and it may have positive effects on blood oxygenation and $\mathrm{CO}_{2}$ clearance. One other study, conducted in the prehospital environment, found that end-tidal $\mathrm{CO}_{2}$ could be useful in diagnosing pulmonary embolism. Three articles addressed disaster medicine, the first of which described a triage system for use during pandemic influenza that demonstrated high reliability in delineating patients with a good chance of survival from those likely to die. The other two studies, both drawn from the 2008 Sichuan earthquake experience, showed success in treating crush injured patients in an on-site tent ICU and, in the second case, how the epidemiology of earthquake injuries and related factors predicted mortality.
\end{abstract}

*Correspondence: Jeffery.Metzger@UTSW.edu

Department of Surgery/Emergency Medicine, University of Texas Southwestern Medical Center at Dallas, 5323 Harry Hines Blvd, Mail Code 8579, Dallas, TX 753908579, USA

\section{Introduction}

Nine papers were published in Critical Care during 2009 on cardiac arrest resuscitation, prehospital medicine, trauma care and disaster response. Five of these articles specifically discussed various issues related to cardiac arrest and cardiopulmonary resuscitation (CPR), including CPR training perspectives, predictors of outcomes (two papers), a recommended approach for the harvesting of more kidneys for transplantation and actions to take with pump-less interventional lung assist devices during CPR conditions. Another article discussed the use of end-tidal $\mathrm{CO}_{2}$ as a method to diagnose pulmonary embolism in the pre-hospital environment and the remaining three of the 2009 papers discussed issues related to trauma and disaster medicine, including the issue of triage for pandemics and medical management of earthquake victims.

\section{Cardiac arrest and cardiopulmonary resuscitation Training CPR at even younger ages?}

It has been recognized for a half century that early bystander-initiated basic CPR may increase the rate of survival following sudden out-of-hospital cardiac arrest almost four-fold, yet the frequency of bystander CPR remains relatively low [1]. In an attempt to increase the number of people trained in CPR and better ensure its subsequent performance, one focus has been the training of children from an early age. Several studies have shown that adolescents are not only physically able to perform $\mathrm{CPR}$, but they are also capable of retaining the information on a long-term basis. Accordingly, many school programs are already in place [2-8]. In their 2009 Critical Care publication, Fleischhackl and colleagues [9] demonstrated that students as young as 9 years of age are able to effectively learn and perform basic CPR. This study was conducted beginning with random selection of 11 schools across four states in Austria. In these schools, a total of 180 students between the ages of 9 and 18 years were trained in CPR, including automated external defibrillator use. Approximately 4 months after training, students in this age range had no difficulty retaining the instructions. Also, age had no independent effect on the physical ability to provide adequate chest compressions 
or rescue ventilation, even in the 9-year-old age group. However, the depth of chest compressions and adequacy of tidal volumes were still influenced the most by the body mass index of the person applying CPR ( $\mathrm{r}=0.21$, $P=0.01$ ). These data suggest that children 9 years old or younger may have some physical difficulty performing the physical aspects of CPR depending on their size, but, cognitively, they still can learn the skills and also begin early psycho-motor training, making them much more prepared as they grow.

\section{Will mild therapeutic hypothermia after cardiac arrest affect S-100 measurements?}

Therapeutic hypothermia has shown significant promise in improving outcomes for cardiac arrest patients who have achieved return of spontaneous circulation (ROSC) $[10,11]$. Despite the improved survival rates, however, there are still a large number of patients who have had such a severe hypoxic insult to their brain that they still inevitably have poor outcomes.

Accordingly, many investigators are seeking an early marker for this type of severe neurologic insult. One biomarker that has shown promise as a very sensitive indicator of poor neurologic recovery after cardiac arrest is the astroglial protein S-100 [12-17]. Several studies have shown the utility of S-100 measurements in predicting brain damage and poor neurologic outcome, but the question then arises as to whether mild therapeutic hypothermia (MTH) can affect S-100 levels and thus its reliability as a predictor under those circumstances. Consequently, Derwall and colleagues, in a 2009 Critical Care publication [18], compared measured S-100 levels in patients who received MTH versus those who remained normothermic after ROSC following nontraumatic out-of-hospital cardiac arrest. Moreover, they examined all presentations of non-traumatic cardiac arrest, not just those presenting with ventricular fibrillation.

Their results showed no difference in S-100 levels in patients receiving $\mathrm{MTH}$ versus those who remained normothermic. In both the $\mathrm{MTH}$ and normothermic groups, they did show the expected elevation in serum S-100 levels in patients with poor neurologic outcomes, not only at baseline, but also at 24 and 72 hours after admission ( $P=0.028,0.002$, and 0.030 , respectively). They also confirmed a strong trend toward higher S-100 levels at 24 hours post-admission for those patients with poor neurologic outcomes who underwent $\mathrm{MTH}$ $(P=0.001)$. The authors concluded that MTH has no detectable influence on S-100 levels in survivors of nontraumatic out-of-hospital cardiac arrest, regardless of presentation or temperature management. The authors point out, however, that while S100 is very sensitive for hypoxic brain injury, it is not very specific and factors such as infection and inflammation, including sepsis, can also elevate serum S-100 levels and may have affected the results of their study.

\section{Another predictor of poor neurologic outcome}

The concept of predicting neurologic outcomes and attempting to quantify neurologic damage by measuring serum levels of neuro-specific proteins is not entirely novel. As discussed previously, patients with more devastating neurologic insults are expected to manifest higher levels of neuron-specific proteins such as S-100 and neuron-specific enolase. However, it is important to note that these severe neurologic insults are generally the result of global (systemic) hypoperfusion and hypoxia. With that perspective, in their 2009 Critical Care article, Hasper and colleagues [19] set out to investigate the relationship between neurologic outcomes and concomitant acute kidney injury. Over a 3-year period, they identified 195 patients who were admitted to the hospital following cardiac arrest resuscitation. In their study, they specifically monitored both serum creatinine levels and neurologic outcomes.

Utilizing the Acute Kidney Injury Network guidelines for acute kidney injury, the researchers not only determined that this complication is very common in cardiac arrest patients (39\%), but also that those with poor neurologic outcomes are affected more frequently. In reviewing the results, one parameter that might be useful in determining outcome in patients after cardiac arrest is the change in serum creatinine over the first 24 hours ( $\Delta$ Crea24 of less than -0.19 predicted a good neurologic outcome with a sensitivity of $63 \%$ and specificity of $71 \%$ ). However, when the creatinine measurements remained unchanged (remained consistently elevated) or rose significantly, the relative risk for an unfavorable neurologic outcome was 2.1. In contrast, when the creatinine declined by $0.2 \mathrm{mg} / \mathrm{dl}$, the relative risk was 0.46 . In summary, this article demonstrated a strong link between an initial decline in serum creatinine level and improved neurologic outcome, while a poorer neurologic outcome was associated with a consistently elevated or rising creatinine.

\section{Pulse-less kidney donors}

With further respect to kidney function, during 2007, in the United States alone, more than 4,000 patients died while waiting for a kidney transplantation [20]. One possible solution to the shortage of 'living-related' donors or brain dead donors (with on-going spontaneous circulation) is to use organs from non-heart-beating donors (NHBDs). Programs using this NHBD technique have already been developed in many western countries [21-26]. Typically, this protocol involves controlled donors for whom life support is being withdrawn and 
when the patient's heart stops and pronouncement of death made, the organs are harvested immediately. In Critical Care in 2009, Fieux and colleagues [27] describe a novel program in which patients with out-of-hospital cardiac arrest received on-going mechanical ventilation and continuous external cardiac massage using an AutoPulse ${ }^{\circ}$ device. In cases in which resuscitation efforts appeared to be futile, after hospital arrival, cardiac massage was held for 5 minutes while death was certified. Once this pronouncement occurred, it was confirmed that the patient was not on the automated National Registry for organ donation refusal. Consent was then obtained from next-of-kin and a double-balloon intraaortic catheter was inserted and a chilled preserving solution infused. Kidneys were then removed surgically and subsequently transplanted into a matched donor.

During the 16-month observation period of the study, the researchers documented that 31 kidneys had been removed from 27 NHBD subjects. Of the 24 graft recipients with follow-up information, only 3 of them failed (1 graft vein thrombosis, 1 immuno-suppression cessation, and 1 primary non-functioning organ). The researchers did note a rate of delayed graft function of $92 \%$, but the overall graft survival rate was $89 \%$ at 3 - and 6-month follow-up assessments. In summary, this study helped to confirm a reasonable strategy for increasing the number of viable kidneys available for transplantation. However, it is important to appreciate that a large part of the success of this program was dependent not only on strict adherence to inclusion and exclusion guidelines, but also prompt harvesting and transplantation times.

\section{Should we clamp interventional lung assist devices during CPR?}

Pump-less interventional lung assist (ILA) devices are mechanical tools that use an extracorporeal membrane oxygenator to help remove carbon dioxide from the blood. These devices take advantage of the patient's arterio-venous pressure gradient to move blood across this membrane; thus, they do not require an active pump. Several studies have shown that these devices are very facile at removing carbon dioxide from the blood [28-31], but, in contrast, their effect on oxygenation appears limited [32-34], primarily because the ILA device is usually actually driven by oxygenated arterial blood (arterio-venous pressure gradient). In that respect, it is not clear whether the device should be clamped or left open if the patient develops cardiac arrest.

In their 2009 study published in Critical Care, Zick and colleagues [35] attempted to address this question with a bench experiment. Specifically, the researchers took 12 pigs, inserted cannulae, and connected them to an ILA device while monitoring several parameters, including mean arterial pressure, end-tidal $\mathrm{PCO}_{2}, \mathrm{PaO}_{2}$ and
$\mathrm{PaCO}_{2}$. They first induced acute lung injury by repeated broncho-alveolar lavage and then induced ventricular fibrillation. Following the cardiac arrest, they clamped the ILA devices in six pigs and left the ILA device open in the other six. They then performed manual chest compressions on the animals for 30 minutes. In the end, the researchers found no differences in the mean arterial pressure or end-tidal $\mathrm{PCO}_{2}$ when the ILA device was either left open or clamped. They did show that the $\mathrm{PaCO}_{2}$ was significantly lower in the group with the ILA system open throughout the study period, whether at 10 minutes $(P<0.05)$, or at 20 and 30 minutes $(P<0.005)$. In general, there was a trend for a higher $\mathrm{PaO}_{2}$, but this finding was only statistically significant at the 20-minute mark $(P<0.05)$. It appears then, from this experimental study, that while leaving an ILA device open during CPR has no harmful effects on arterial pressure, it may have some beneficial effects on both $\mathrm{PaO}_{2}$ and $\mathrm{PaCO}_{2}$. The authors concluded that if a patient with an ILA device in place experiences cardiac arrest, the ILA device should be kept open during the resuscitation efforts.

It is worth mentioning that the above model of lung injury in swine may have some key differences from lung injury in humans. Swine lack alveolar collateral ventilation, which has been shown in humans, which may affect gas exchange differently in this model [36]. In addition, pigs have been shown to have a smaller functional lung unit, which may lead to higher levels of pulmonary hypertension with lung injury, also leading to differing hemodynamics and gas exchange than in human models, though the significance of this difference is unknown [37].

\section{Pulmonary embolism}

Each year, pulmonary embolism (PE) has been reported to affect between 23 and 69 people per 100,000 population, with a mortality rate of approximately 15 to $18 \%$ [38-41]. The often elusive diagnosis and/or exclusion of PE has been the subject of many studies and review articles. Laboratory tests such as the D-dimer can be reasonably helpful at ruling out PE if the test result is negative, but it is extremely non-specific when positive [42]. Also, this test is of little utility in trauma patients, as most of these patients will have had some degree of injury-induced micro-vascular trauma or the residua of surgical intervention. In order to improve predictive capabilities, attempts at clinical prediction rules using more than one clinical factor have been established to help to better determine a likelihood of PE [43]. Among the clinical factors is the use of end-tidal $\mathrm{CO}_{2}\left(\mathrm{PetCO}_{2}\right)$ measurements. Studies have shown that as pulmonary embolism blocks returning venous blood flow to a particular area of a lung, it creates a high-ventilation to low-perfusion state in that zone, thus decreasing the 
amount of $\mathrm{CO}_{2}$ that can be eliminated across those gas exchange areas and, at times, across much of the entire lung $[44,45]$.

In a 2009 study by Rumpf and colleagues in Critical Care [46], prehospital care providers used a combination of clinical predictors - a D-dimer test and $\mathrm{PetCO}_{2}$ - to exclude or confirm PE in the prehospital setting. They showed that in patients with a positive D-dimer, but low clinical probability of PE using the Wells criteria, a $\mathrm{PetCO}_{2}$ reading of more than $28 \mathrm{mmHg}$ is a potentially safe method for excluding PE with a sensitivity of $100 \%$ (95\% confidence interval $(\mathrm{CI})=89$ to $100 \%)$. In fact, a PetCO $_{2}$ above $28 \mathrm{mmHg}$ alone excluded PE with a sensitivity of $92.6 \%$. Conversely, patients with a positive D-dimer, a high clinical probability of $\mathrm{PE}$, and a $\mathrm{PetCO}_{2}$ below $28 \mathrm{mmHg}$ had a specificity for PE of $93.2 \%$, although the sensitivity was only $58.5 \%$ ( $95 \% \mathrm{CI}=79$ to $98 \%)$. In turn, the negative predictive value of this combination-predictor was $94.2 \%$ (95\% CI $=83$ to $99 \%)$ with a specificity of $83 \%(95 \% \mathrm{CI}=71$ to $91 \%)$, and a positive predictive value of $79.2 \%$ ( $95 \% \mathrm{CI}=65$ to $89 \%$ ). The conclusion was that $\mathrm{PetCO}_{2}$ may be a useful adjunct in the identification or exclusion of PE.

\section{Disaster medicine}

\section{Triage for a pandemic}

In April 2009, the US Centers for Disease Control and Prevention reported a novel strain of H1N1 influenza virus in two children [47]. By June 2009, the World Health Organization had declared a worldwide influenza pandemic [48]. As a result the critical care management of pandemics came to center stage. Though this particular pandemic proved, at least for the time-being, to be relatively mild in terms of its eventual impact, the concern over eventual large-scale world-wide deaths from a pandemic is still a major consideration for critical care practitioners. To mitigate the effects of a worldwide pandemic that might lead to extremely large numbers of patients requiring intensive medical care would require actions and preparations such as expanding ICU capacity, developing management systems to control resources, and ensuring adequate stockpiles of ventilators, medications, equipment, and supplies [49]. It had been recognized well before this recent influenza pandemic that despite the development of enhanced surge capacity and stockpiling supplies and equipment, the demand for these resources may exceed supply in some cases. At that point in time, a triage process must be implemented to provide fair and efficient distribution of resources that would ensure the most benefit to the community as a whole.

In 2004, a group of clinicians and public health specialists convened at the request of the steering committee of the Ontario Health Plan for an Influenza
Pandemic to develop a triage protocol for critical care patients during an influenza pandemic [50]. The protocol that they developed is based on the Sequential Organ Failure Assessment (SOFA) score, which can be subcategorized into its four main components: inclusion criteria; exclusion criteria; minimum qualifications for survival; and a prioritization tool. The inclusion criteria established were intended to identify patients that are sick enough to require intensive care. The exclusion criteria, however, would eliminate those patients who have a poor prognosis even in the best of circumstances, who would require resources that cannot be provided during a pandemic, and who have advanced underlying medical illness that carries a poor prognosis, even without their current concomitant illness. The 'minimum qualifications for survival' (MQS) represent a ceiling on the amount of resources that can be expended on an individual and this determination required a reassessment at 48 and 120 hours. The final step involved the prioritization of patients using the familiar green, yellow, red, and blue (or black) color scheme. The protocol underwent multiple periods of review and revision, but had yet to be tested.

Accordingly, in an article published in Critical Care in 2009, Christian and colleagues tested the Ontario triage system by applying it hypothetically to a cohort of 234 patients admitted to two ICU facilities during an 8-week period of peak occupancy [51]. They found that, of these 234 patients, 175 of them met inclusion criteria upon admission and another 3 met the inclusion criteria during their hospital admission. Of these 178,115 patients (65\%) did not meet the exclusion criteria. These patients were further monitored and prioritized on day 2 and 5 to determine if they later met the exclusion criteria or the MQS criteria and 85 patients still eluded these criteria. In the final analysis of the entire cohort, $39.7 \%$ met the triage exclusion criteria or MQS and would have been managed expectantly, meaning they would have been given only comfort care while diverting the other critical care resources to those with a higher likelihood of survival.

As the primary outcome measure for this study was the utility of the triage protocol, it was important to note that the triage officers were confident or very confident in $68.4 \%$ of their scores and that arbitration was required in $54.9 \%$ of cases. When looking at the decrease in resource utilization, they determined that application of the triage protocol would have reduced the number of ventilator days by $49.3 \%$ and the number of ICU days by $52.6 \%$. The survival rate for patients in the red and yellow categories (93.7\% and $62.5 \%)$ were significantly higher than the survival rate in the blue category on admission (24.6\%). Therefore, the authors believed that this protocol generally was able to identify those patients with a higher likelihood of survival. 


\section{Earthquake response}

At 2:28 p.m. on 12 May 2008 an earthquake measuring 8.0 on the Richter scale shook the Wenchuan region of Sichuan province in southwest China. Over 69,000 people were killed and over 300,000 injured. While direct trauma to major organs such as the brain or heart is a major cause of death in earthquakes and building collapses, crush injuries can also be a significant source of morbidity and mortality. Crush injuries should undergo immediate and aggressive treatment to prevent progression to crush syndrome and it is therefore a relative priority to treat those who have progressed to that state [52]. In this particular earthquake scenario in China, however, rescue efforts were hampered by the mountainous terrain and damaged roads [53]. Therefore, in their article, Li and colleagues [53] describe relevant cases of patients seen in a front-line tent ICU that was set up by a rescue team. This tent ICU was established on the site of the disaster, allowing for prompt treatment and not requiring prolonged and dangerous transportation during evacuation. The major treatments afforded to these patients included anti-shock treatment, surgical intervention, and hemodialysis. During a 14-day period, 32 patients were admitted to this tent ICU; 17 of the total 32 patients (53\%) met the diagnostic criteria for crush syndrome and six of these patients died. All six of the dying patents had serum creatinine kinase levels of over $5,000 \mu / \mathrm{L}$. The authors concluded that close monitoring and prompt intervention helped to save the lives of the 26 remaining patients.

\section{Characterizing earthquake casualties}

Despite the difficult logistics confounding the earthquake rescue efforts in Wenchuan, some patients could be evacuated and the hospital receiving the largest number of these patients was the West China Hospital, located approximately 50 miles away [54]. In another related article published during 2009 in Critical Care, Wen and colleagues [54] described a hospital-based, case-control study of all the deaths due to earthquake injuries, using earthquake survivors as the controls. The researchers determined that severe traumatic brain injury, infection, multiple system organ failure, and prior major disease were the significant determinants of earthquake-related deaths and that patients with severe traumatic brain injury had the greatest risk of death (odds ratio $=253.3$, $95 \% \mathrm{CI}=8.9$ to 7208.6 ). Consistent with the conclusions of the previous on-site ICU study, they also found that local hospital treatment (prior to transfer to the study hospital) lowered the risk of earthquake-related death (odds ratio $=0.4,95 \%$ CI 0.2 to 0.9 ).

\section{Conclusions}

The 2009 volume of Critical Care contained several articles related to resuscitation, prehospital medicine, trauma and disaster medicine. While it is generally accepted that increasing the number of people trained in CPR may help improve the frequency of bystander CPR, it is now well-accepted that school children may be a valuable target audience to teach $[9,55]$. In one of the 2009 Critical Care articles, it was shown that children as young as 9 years of age are able to cognitively learn the skills and, if they are unable to physically perform them due to size at the time of instruction, they will be better prepared to perform CPR in the future. For those patients who do survive to the hospital and get ROSC, the astroglial protein predicts a poor neurological outcome. In now appears, according to one of the 2009 papers, that use of mild therapeutic hypothermia does not change that observation. Another investigation demonstrated that one method to help predict neurologic outcomes is measuring renal function, specifically the change in creatinine over the first 24 hours. Moreover, a drop in creatinine of at least $0.2 \mathrm{mg} / \mathrm{dl}$ suggests a much higher likelihood of a good neurologic outcome. With further respect to kidney function and cardiac arrest, another study demonstrated that, for those patients for whom CPR is unsuccessful, a certain subset still may be a viable source of donor kidneys for transplantation. In an experimental model of cardiac arrest published in the 2009 volume, it was shown that when an ILA device is in place, there may be some benefit to leaving the device open (instead of clamping it) during CPR efforts. In another article, it was also shown that a $P$ etCO $\mathrm{O}_{2}$ reading of more than $28 \mathrm{mmHg}$ may be useful in excluding the presence of a significant PE, even in patients with a positive D-dimer measurement. Reports from the 2009 volume also suggest that a triage tool developed for pandemics may be useful in separating patients with a good chance of survival from those with a poor prognosis and that, following an earthquake, there may be significant success when treating crush injured patients in an on-site tent ICU and that the presence of severe head injuries was the greatest predictor of mortality.

Competing interests

The authors declare that they have no competing interests.

\section{Abbreviations}

$\mathrm{Cl}$, confidence interval: $\mathrm{CPR}$, cardiopulmonary resuscitation; ILA, interventional lung assist; MQS, minimum qualifications for survival; MTH, mild therapeutic hypothermia; NHBD, non-heart-beating donors; PE, pulmonary embolism; PetCO ${ }_{2}$, end-tidal $\mathrm{CO}_{2}$; $\mathrm{ROSC}$, return of spontaneous circulation.

\section{Published: 5 November 2010}

References

1. Vaillancourt C, Stiell IG, Wells GA: Understanding and improving low bystander CPR rates: a systematic review of the literature. CJEM 2008, 10:51-65.

2. Lafferty C, Larsen P, Galletly D: Resuscitation teaching in New Zealand schools. NZMed J 2003, 116:U582.

3. Vanderschmidt H, Burnap TK, Thwaites JK: Evaluation of a cardiopulmonary resuscitation course for secondary schools retention study. Med Care 1976, 
$14: 181-184$

4. Lewis RM, Fulstow R, Smith GB: The teaching of cardiopulmonary resuscitation in schools in Hampshire. Resuscitation 1997, 35:27-31.

5. Liberman M, Golberg N, Mulder D, Sampalis J: Teaching cardiopulmonary resuscitation to CEGEP students in Quebec - a pilot project. Resuscitation 2000, 47:249-257.

6. Lester CA, Weston CF, Donnelly PD, Assar D, Morgan MJ: The need for wider dissemination of CPR skills: are schools the answer? Resuscitation 1994, 28:233-237.

7. Lubrano R, Romero S, Scoppi P, Cocchi G, Baroncini S, Elli M, Turbacci M, Scateni S, Travasso E, Benedetti R, Cristaldi S, Moscatelli R: How to become an under 11 rescuer: a practical method to teach first aid to primary schoolchildren. Resuscitation 2005, 64:303-307.

8. Reder S, Quan L: Cardiopulmonary resuscitation training in Washington state public high schools. Resuscitation 2003, 56:283-288.

9. Fleischhackl R, Nuernberger A, Sterz F, Schoenberg C, Urso T, Habart T, Mittlboeck M, Chandra-Strobos N: School children sufficiently apply life supporting first aid: a prospective investigation. Crit Care 2009, 13:R127.

10. Bernard SA, Gray TW, Buist MD, Jones BM, Silvester W, Gutteridge G, Smith K: Treatment of comatose survivors of out-of-hospital cardiac arrest with induced hypothermia. N Engl J Med 2002, 346:557-563.

11. Hypothermia after Cardiac Arrest Study G: Mild therapeutic hypothermia to improve the neurologic outcome after cardiac arrest. N Engl J Med 2002, 346:549-556. A published erratum appears in N Engl J Med 2002, 346:1756.

12. Bottiger B, Mobes S, Glatzer R, Bauer H, Gries A, Bartsch P, Motsch J, Martin E: Astroglial protein S-100 is an early and sensitive marker of hypoxic brain damage and outcome after cardiac arrest in humans. Circulation 2001, 103:2694-2698.

13. Ekmektzoglou K, Xanthos T, Papadimitriou L: Biochemical markers (NSE, S-100, IL-8) as predictors of neurological outcome in patients after cardiac arrest and return of spontaneous circulation. Resuscitation 2007, 75:219-228

14. Pfeifer R, Borner A, Krack A, Sigusch H, Surber R, Figulla H: Outcome after cardiac arrest: predictive values and limitations of the neuroproteins neuron-specific enolase and protein S-100 and the Glasgow Coma Scale. Resuscitation 2005, 65:49-55.

15. Hachimi-Idrissi S, Auwera M, Schiettecatte J, Ebinger G, Michotte Y, Huyghens L: S-100 protein as early predictor of regaining consciousness after out of hospital cardiac arrest. Resuscitation 2002, 53:251-257.

16. Shinozaki K, Oda S, Sadahiro T, Nakamura M, Abe R, Nakada TA, Nomura F, Nakanishi K, Kitamura N, Hirasawa H: Serum S-100B is superior to neuronspecific enolase as an early prognostic biomarker for neurological outcome following cardiopulmonary resuscitation. Resuscitation 2009, 80:870-875.

17. Rosen H, Sunnerhagen K, Herlitz J, Blomstrand C, Rosengren L: Serum levels of the brain-derived proteins S-100 and NSE predict long-term outcome after cardiac arrest. Resuscitation 2001, 49:183-191.

18. Derwall M, Stoppe C, Brucken D, Rossaint R, Fries M: Changes in S-100 protein serum levels in survivors of out-of-hospital cardiac arrest treated with mild therapeutic hypothermia: a prospective, observational study. Crit Care 2009, 13:R58.

19. Hasper D, von Haehling S, Storm C, Jorres A, Schefold J: Changes in serum creatinine in the first 24 hours after cardiac arrest indicate prognosis: an observational cohort study. Crit Care 2009, 13:R168.

20. Delmonico FL, McBride MA: Analysis of the wait list and deaths among candidates waiting for a kidney transplant. Transplantation 2008, 86:1678-1683

21. Abelha F, Botelho M, Fernandes V, Barros H: Determinants of postoperative acute kidney injury. Crit Care 2009, 13:R79

22. Kootstra G: Expanding the donor pool: the challenge of non-heart-beating donor kidneys. Transplant Proc 1997, 29:3620.

23. Sanchez-Fructuoso A: Kidney transplantation from non-heart-beating donors. Transplant Proc 2007, 39:2065-2067.

24. Weber M, Dindo D, Demartines N, Ambuhl P, Clavien P: Kidney transplantation from donors without a heartbeat. N Engl J Med 2002, 347:248-255

25. Metcalfe M, Butterworth P, White S, Saunders R, Murphy G, Taub N, Veitch P, Nicholson M: A case-control comparison of the results of renal transplantation from heart-beating and non-heart-beating donors. Transplantation 2001, 71:1556-1559.

26. Yoshida K, Endo T, Saito T, Iwamura M, Ikeda M, Kamata K, Sato K, Baba S:
Factors contributing to long graft survival in non-heart-beating cadaveric renal transplantation in Japan: a single-center study at Kitasato University. Clin Transplant 2002, 16:397-404.

27. Fieux F, Losser MR, Bourgeois E, Bonnet F, Marie O, Gaudez F, Abboud I, Donay $J$, Roussin F, Mourey F, Adnet F, Jacob L: Kidney retrieval after sudden out of hospital refractory cardiac arrest: a cohort of uncontrolled non heart beating donors. Crit Care 2009, 13:R141.

28. Conrad S, Brown E, Grier L, Baier J, Blount J, Heming T, Zwischenberger J, Bidani A: Arteriovenous extracorporeal carbon dioxide removal: a mathematical model and experimental evaluation. ASAIO J 1998, 44:267-277.

29. Conrad S, Zwischenberger J, Grier L, Alpard S, Bidani A: Total extracorporeal arteriovenous carbon dioxide removal in acute respiratory failure: a phase I clinical study. Intensive Care Med 2001, 27:1340-1351.

30. Zwischenberger J, Conrad S, Alpard S, Grier L, Bidani A: Percutaneous extracorporeal arteriovenous $\mathrm{CO} 2$ removal for severe respiratory failure. Ann Thorac Surg 1999, 68:181-187.

31. Frank B, Tao W, Brunston R, Alpard S, Bidani A, Zwischenberger J: High flow/ low resistance cannulas for percutaneous arteriovenous carbon dioxide removal. ASAIO J 1997, 43:M817-820.

32. Bein T, Prasser C, Philipp A, Muller T, Weber F, Schlitt H, Schmid F, Taeger K, Birnbaum D: [Pumpless extracorporeal lung assist using arterio-venous shunt in severe ARDS. Experience with 30 cases]. Anaesthesist 2004, 53:813-819.

33. Liebold A, Reng C, Philipp A, Pfeifer M, Birnbaum D: Pumpless extracorporeal lung assist - experience with the first 20 cases. Eur J Cardiothorac Surg 2000 17:608-613.

34. Reng M, Philipp A, Kaiser M, Pfeifer M, Gruene S, Schoelmerich J: Pumpless extracorporeal lung assist and adult respiratory distress syndrome. Lancet 2000, 356:219-220

35. Zick G, Schadler D, Elke G, Pulletz S, Bein B, Scholz J, Frerichs I, Weiler N: Effects of interventional lung assist on haemodynamics and gas exchange in cardiopulmonary resuscitation: a prospective experimental study on animals with acute respiratory distress syndrome. Crit Care 2009, 13:R17.

36. Kuriyama T, Latham LP, Horwitz LD, Reeves JT, Wagner WW Jr: Role of collateral ventilation in ventilation-perfusion balance. J App/ Physio/ 1984, 56:1500-1506.

37. Hedenstierna G, Hammond M, Mathieu-Costello O, Wagner PD: Functional lung unit in the pig. Respir Physiol 2000, 120:139-149.

38. Anderson FA Jr, Wheeler HB, Goldberg RJ, Hosmer DW, Patwardhan NA, Jovanovic B, Forcier A, Dalen JE: A population-based perspective of the hospital incidence and case-fatality rates of deep vein thrombosis and pulmonary embolism: the Worcester DVT Study. Arch Intern Med 1991, 151:933-938.

39. Oger E: Incidence of venous thromboembolism: a community-based study in Western France. EPI-GETBP Study Group. Groupe d'Etude de la Thrombose de Bretagne Occidentale. Thrombosis Haemostasis 2000, 83:657-660

40. Silverstein MD, Heit JA, Mohr DN, Petterson TM, O'Fallon WM, Melton III L. Trends in the incidence of deep vein thrombosis and pulmonary embolism: a 25-year population-based study. Arch Intern Med 1998 158:585-593.

41. Goldhaber S, Visani L, De Rosa M: Acute pulmonary embolism: clinical outcomes in the International Cooperative Pulmonary Embolism Registry (ICOPER). Lancet 1999, 353:1386-1389.

42. Stein P, Hull R, Patel K, Olson R, Ghali W, Brant R, Biel R, Bharadia V, Kalra N: D-dimer for the exclusion of acute venous thrombosis and pulmonary embolism: a systematic review. Ann Intern Med 2004, 140:589-602.

43. Wells PS, Anderson DR, Rodger M, Ginsberg JS, Kearon C, Gent M, Turpie AG, Bormanis J, Weitz J, Chamberlain M, Bowie D, Barnes D, Hirsh J: Derivation of a simple clinical model to categorize patients probability of pulmonary embolism: increasing the models utility with SimpliRED D-dimer. Thromb Haemost 2000, 83:416-420.

44. Robin E, Julian D, Travis D, Crump C: A physiologic approach to the diagnosis of acute pulmonary embolism. N Engl J Med 1959, 260:586-591.

45. Burki N: The dead space to tidal volume ratio in the diagnosis of pulmonary embolism. Am Rev Respir Dis 1986, 133:679-685.

46. Rumpf T, Krizmaric M, Grmec S: Capnometry in suspected pulmonary embolism with positive D-dimer in the field. Crit Care 2009, 13:R196.

47. Centers for Disease C, Prevention: Swine influenza A (H1N1) infection in two children - Southern California, March-April 2009. MMWR Morb Mortal Wkly 
Rep 2009, 58:400-402.

48. Zarocostas J: World Health Organization declares A (H1N1) influenza pandemic. BMJ 2009, 338:b2425.

49. Sprung CL, Zimmerman JL, Christian MD, Joynt GM, Hick JL, Taylor B, Richards GA, Sandrock C, Cohen R, Adini B; European Society of Intensive Care Medicine Task Force for Intensive Care Unit Triage during an Influenza Epidemic or Mass Disaster: Recommendations for intensive care unit and hospital preparations for an influenza epidemic or mass disaster: summary report of the European Society of Intensive Care Medicine's Task Force for intensive care unit triage during an influenza epidemic or mass disaster. Intensive Care Med, 36:428-443.

50. Christian M, Hawryluck L, Wax R, CookT, Lazar N, Herridge M, Muller M, Gowans D, Fortier W, Burkle F: Development of a triage protocol for critical care during an influenza pandemic. CMAJ 2006, 175:1377-1381.

51. Christian M, Hamielec C, Lazar N, Wax R, Griffith L, Herridge M, Lee D, Cook D: A retrospective cohort pilot study to evaluate a triage tool for use in a pandemic. Crit Care 2009, 13:R170

52. Smith J, Greaves I: Crush injury and crush syndrome: a review. J Trauma 2003, 54(5 Suppl):S226-230

53. Li W, Qian J, Liu X, Zhang Q, Wang L, Chen D, Lin Z: Management of severe crush injury in a front-line tent ICU after 2008 Wenchuan earthquake in China: an experience with 32 cases. Crit Care 2009, 13:R178.

54. Wen J, Shi YK, Li YP, Wang L, Cheng L, Gao Z, Li L: Risk factors of earthquake inpatient death: a case control study. Crit Care 2009, 13:R24.

55. Roppolo LP, Pepe PE: Retention, retention, retention: targeting the young in CPR skills training! Crit Care 2009, 13:185

doi:10.1186/cc9302

Cite this article as: Metzger JC, et al: Year in review 2009: Critical care cardiac arrest, trauma and disasters. Critical Care 2010, 14:242. 